\title{
Nuclear fragmentation: sampling the instabilities of binary systems
}

\author{
V. $\operatorname{Baran}^{a b)}$, M. Colonna ${ }^{a)}$, M. Di Toro ${ }^{a)}$, V. Greco ${ }^{a)}$ \\ a) Laboratori Nazionali del Sud, Via S. Sofia 44, I-95123 Catania, Italy and University of Catania \\ b) NIPNE-HH, Bucharest, Romania
}

(November 10, 2018)

\begin{abstract}
We derive stability conditions of Asymmetric Nuclear Matter $(A N M)$ and discuss the relation to mechanical and chemical instabilities of general two-component systems. We show that the chemical instability may appear as an instability of the system against isoscalar-like rather than isovector-like fluctuations if the interaction between the two constituent species has an attractive character as in the case of $A N M$. This leads to a new kind of liquid-gas phase transition, of interest for fragmentation experiments with radioactive beams.
\end{abstract}

PACS numbers: 21.65.+f, 25.70.Pq, 71.10.Ay

A binary system manifests a richer thermodynamical behaviour as a consequence of a new parameter, the concentration, which is required for a complete description of its states. The phase transitions are more complex because they have to accommodate one more conservation law. A thermodynamical state can be unstable not only mechanically but also chemically.

Nuclear matter belongs to this class of systems. The process of multifragmentation following the collision of heavy nuclei at medium energies is expected to show features analogous to usual liquid-gas phase transitions of water [1] 3]. In particular, the spinodal decomposition appears to be an important mechanism leading to phase separation in symmetric nuclear matter $(S N M)$ [ [3] 6 , as confirmed in recent experiments [7]. The relevance of instabilities in $A N M$-fragmentation has been already discussed few years ago [8 10]. It was shown that in these systems a kind of diffusive (or chemical) spinodal rather than the mechanical spinodal is significant. However the detailed nature of fluctuations responsible for such instability has not been clarified yet. This is a quite important aspect since, as we will see, it provides reliable information on the character of the interaction in the medium. We remark that the effect is leading to the "isospin distillation" that can be experimentally observed, see the recent data 11].

In one-component systems the mechanical instability is related to instability against density fluctuations as the result of the strong attraction between constituents. In symmetric binary systems, like $S N M$, one encounters two kinds of density fluctuations: i) isoscalar, when the densities of the two components oscillate in phase with equal amplitude, ii) isovector when the two densities fluctuate still with equal amplitude but out of phase. Mechanical instability is associated with insta- bility against isoscalar fluctuations leading to cluster formation while chemical instability is related to instability against isovector fluctuations, leading to species separation. We will show that in asymmetric binary systems, as in $A N M$, this direct correspondence between the nature of fluctuations and the thermodynamical instability is lost.

An appropriate framework for this investigation is provided by the Fermi liquid theory which has been already applied to the study of instabilities in symmetric binary systems as $S N M$ (the two components being protons and neutrons) and the liquid ${ }^{3} \mathrm{He}$ (spin-up and spin-down components) 12,13.

We first investigate the thermodynamical stability of $A N M$ at $T=0$ extending to the asymmetric case the formalism introduced in 12. The distribution functions for protons and neutrons are:

$$
f_{q}^{(0)}\left(\epsilon_{p}^{q}\right)=\Theta\left(\mu_{q}-\epsilon_{p}^{q}\right), \quad q=n, p
$$

where $\mu_{q}$ are the corresponding chemical potentials. The nucleon interaction is characterized by the Landau parameters:

$$
F^{q_{1} q_{2}}=N_{q_{1}} V^{2} \frac{\delta^{2} \mathcal{H}}{\delta f_{q_{1}} \delta f_{q_{2}}}, \quad N_{q}(T)=\int \frac{-2 d \mathbf{p}}{(2 \pi \hbar)^{3}} \frac{\partial f_{q}(T)}{\partial \epsilon_{p}^{q}}
$$

where $\mathcal{H}$ is the energy density, $\mathrm{V}$ is the volume and $N_{q}$ is the single-particle level density at the Fermi energy. At $T=0$ this reduces to $N_{q}(0)=m p_{F, q} /\left(\pi^{2} \hbar^{3}\right)$, were $p_{F, q}$ is the Fermi momentum of the $q$-component. Thermodynamical stability for $T=0$ requires the ground state energy to be an absolute minimum for the undistorted distribution functions, such that the relation:

$$
\delta \mathcal{H}-\mu_{p} \delta \rho_{p}-\mu_{n} \delta \rho_{n}>0
$$

is satisfied when we deform proton and neutron Fermi seas. The distorted distribution functions can be written as $f_{q}(\mathbf{p})=\Theta\left(\epsilon_{F, q}(\theta)-\epsilon_{p}^{q}\right)$, where $\epsilon_{F, q}(\theta)$ is a direction dependent Fermi energy characterizing the distortion. We will follow the usual multipole expansion for the variation

$$
\left[\delta \epsilon_{F, q}(\theta)\right]=\epsilon_{F, q}(\theta)-\mu_{q}=\sum \nu_{q}^{l} P_{l}(\cos \theta)
$$

and for the Landau parameters 


$$
F^{q_{1} q_{2}}\left(\theta_{1}-\theta_{2}\right)=\sum_{l} F_{l}^{q_{1} q_{2}} P_{l}\left(\cos \left(\theta_{1}-\theta_{2}\right)\right) .
$$

We limit ourselves to monopolar deformations considering here momentum independent interactions such that $F_{l=0}^{q_{1} q_{2}}$ are the only non-zero Landau parameters [16. Then, up to second order in the variations, Eq.(3) becomes

$$
\delta \mathcal{H}-\mu_{p} \delta \rho_{p}-\mu_{n} \delta \rho_{n}=\frac{1}{2}\left(a \nu_{p}^{2}+b \nu_{n}^{2}+c \nu_{p} \nu_{n}\right)
$$

where $\nu_{n, p} \equiv \nu_{n, p}^{0}$ and

$$
\begin{gathered}
a=N_{p}(0)\left(1+F_{0}^{p p}\right) ; \quad b=N_{n}(0)\left(1+F_{0}^{n n}\right) \\
\quad c=N_{n}(0) F_{0}^{p n}+N_{p}(0) F_{0}^{n p}=2 N_{n}(0) F_{0}^{p n} .
\end{gathered}
$$

We diagonalize the r.h.s. of Eq.(6) by introducing the following transformation:

$$
\begin{aligned}
& u=\cos \beta \nu_{p}+\sin \beta \nu_{n}, \\
& v=-\sin \beta \nu_{p}+\cos \beta \nu_{n},
\end{aligned}
$$

where the mixing angle $0 \leq \beta \leq \pi / 2$ is defined by

$$
\operatorname{tg} 2 \beta=\frac{c}{a-b}=\frac{N_{n}(0) F_{0}^{p n}+N_{p}(0) F_{0}^{n p}}{N_{p}(0)\left(1+F_{0}^{p p}\right)-N_{n}(0)\left(1+F_{0}^{n n}\right)} .
$$

Then Eq.(6) takes the form

$$
\delta \mathcal{H}-\mu_{p} \delta \rho_{p}-\mu_{n} \delta \rho_{n}=X u^{2}+Y v^{2}
$$

where

$$
\begin{aligned}
X & =\frac{1}{2}\left(a+b+\operatorname{sign}(c) \sqrt{(a-b)^{2}+c^{2}}\right) \\
& \equiv \frac{N_{p}(0)+N_{n}(0)}{2}\left(1+F_{0 g}^{s}\right)
\end{aligned}
$$

and

$$
\begin{aligned}
Y & =\frac{1}{2}\left(a+b-\operatorname{sign}(c) \sqrt{(a-b)^{2}+c^{2}}\right) \\
& \equiv \frac{N_{p}(0)+N_{n}(0)}{2}\left(1+F_{0 g}^{a}\right) .
\end{aligned}
$$

Thanks to the rotation Eq.(8) we separate the total variation Eq.(3) into two independent contributions, the "normal" modes, characterized by the "mixing angle" $\beta$, which depends on the density of states and the details of the interaction.

In the symmetric case, $N_{p}=N_{n} \equiv N, F_{0}^{n n}=F_{0}^{p p}$ and $F_{0}^{n p}=F_{0}^{p n}$, Eq.(6) reduces to

$$
\begin{aligned}
\delta \mathcal{H}-\mu_{p} \delta \rho_{p}-\mu_{n} \delta \rho_{n} & =\frac{N(0)}{2}\left(1+F_{0}^{s}\right)\left(\nu_{p}+\nu_{n}\right)^{2} \\
& +\frac{N(0)}{2}\left(1+F_{0}^{a}\right)\left(\nu_{p}-\nu_{n}\right)^{2}
\end{aligned}
$$

where $F_{0}^{s} \equiv F_{0}^{n n}+F_{0}^{n p}$ and $F_{0}^{a} \equiv F_{0}^{n n}-F_{0}^{n p}$ are symmetric and antisymmetric (or isoscalar and isovector) Landau parameters, and we recover the usual Pomeranchuk stability conditions for pure isoscalar/isovector fluctuations [10].

In the general case we interpret $u$ - and $v$-variations as new independent isoscalar-like and isovector-like fluctuations appropriate for asymmetric systems. The proton and neutron densities will fluctuate in phase for isoscalarlike variations and out of phase for isovector-like variations, see Eq.(8). Moreover, $F_{0 g}^{s}$ and $F_{0 g}^{a}$, defined by Eq. (11,12), can be considered as generalized symmetric and antisymmetric Landau parameters.

From Eq. 10) we see that thermodynamical stability requires $X>0$ and $Y>0$. Equivalently, the following conditions have to be fulfilled:

$$
1+F_{0 g}^{s}>0 \quad \text { and } \quad 1+F_{0 g}^{a}>0
$$

They represent Pomeranchuk stability conditions extended to asymmetric binary systems.

The new stability conditions, Eq.(14), are equivalent to mechanical and chemical stability of a thermodynamical state, [14], i.e.

$$
\left(\frac{\partial P}{\partial \rho}\right)_{T, y}>0 \text { and }\left(\frac{\partial \mu_{p}}{\partial y}\right)_{T, P}>0
$$

where $P$ is the pressure and $y$ the proton fraction, as it can be proved by observing that 18 :

$$
\begin{aligned}
X Y & =N_{p}(0) N_{n}(0)\left[\left(1+F_{0}^{n n}\right)\left(1+F_{0}^{p p}\right)-F_{0}^{n p} F_{0}^{p n}\right] \\
& =\frac{\left(N_{p}(0) N_{n}(0)\right)^{2}}{(1-y) \rho^{2}}\left(\frac{\partial P}{\partial \rho}\right)_{T, y}\left(\frac{\partial \mu_{p}}{\partial y}\right)_{T, P}
\end{aligned}
$$

and:

$$
\begin{gathered}
\left(\frac{\partial P}{\partial \rho}\right)_{T, y}=\frac{\rho y(1-y)}{N_{p}(0) N_{n}(0)}\left(t a+\frac{1}{t} b+c\right) \\
\propto X\left(\sqrt{t} \cos \beta+\frac{1}{\sqrt{t}} \sin \beta\right)^{2}+Y\left(\sqrt{t} \sin \beta-\frac{1}{\sqrt{t}} \cos \beta\right)^{2} \\
\text { with } \quad t=\frac{y}{1-y} \frac{N_{n}(0)}{N_{p}(0)} .
\end{gathered}
$$

From Eq.s(10,14), we are led to define as isoscalar instability the case when the state is unstable against isoscalar-like fluctuations, i.e. when $1+F_{0 g}^{s}<0$ (or $X<0)$. Analogously we deal with isovector instability when the system is unstable against isovector-like fluctuations i.e. when $1+F_{0 g}^{a}<0$ (or $\left.Y<0\right)$.

One can easily see that in symmetric nuclear matter the isoscalar instability, $(X<0, Y>0)$, appears as mechanical instability and the isovector instability, $(X>0, Y<0)$, as chemical instability. Indeed now $t=1, a=b, \beta=\pi / 4$ and so $1+F_{0 g}^{s}($ or $X)$ and 
$\left(\frac{\partial P}{\partial \rho}\right)_{T, y}$ are proportional (see Eq.(17)). This is not true any longer for an asymmetric system.

To simplify the analysis let us assume for the moment that the quantities $a$ and $b$ remain positive in the density range we discuss here and so we can study the effect of the interaction between the two components, given by $c$, on the instabilities of the mixture. This is indeed the case of nuclear matter.

If $c<0$, i.e. for an attractive interaction between the two components, from Eq.(12) we see that the system is stable against isovector-like fluctuations but it becomes isoscalar unstable if $c<-2 \sqrt{a b}$ (see Eq.(11)). However thermodynamically this instability against isoscalarlike fluctuations will show up as a chemical instability if $(-t a-b / t)<c<-2 \sqrt{a b}$ or as a mechanical instability if $c<(-t a-b / t)<-2 \sqrt{a b}$ (see Eq.(17)). Therefore isoscalar-like instabilities are not necessarily equivalent to mechanical instabilities and may manifest instead as chemical instabilities.

If $c>0$, i.e. when the interaction between the components is repulsive, the thermodynamical state is always stable against isoscalar-like fluctuation, but can be isovector unstable if $c>2 \sqrt{a b}$. Since the system is mechanically stable $(a, b, c>0$, see Eq. (17)), the isovector instability is now associated with chemical instability. Such situation will lead to a component separation of the liquid mixture.

Following this line a complete analysis of the instabilities of any binary system can be performed, in connection to signs, strengths and density dependence of the interactions.

We show now a quantitative calculation for asymmetric nuclear matter which illustrates the previous general discussion. We investigate the instabilities of $A N M$ characterized by a potential energy density of Skyrme-like type, [10]

$$
\begin{aligned}
\mathcal{H}_{\text {pot }}\left(\rho_{n}, \rho_{p}\right) & =\frac{A}{2} \frac{\left(\rho_{n}+\rho_{p}\right)^{2}}{\rho_{0}}+\frac{B}{\alpha+2} \frac{\left(\rho_{n}+\rho_{p}\right)^{\alpha+2}}{\rho_{0}^{\alpha+1}} \\
& +\left(C_{1}-C_{2}\left(\frac{\rho}{\rho_{0}}\right)^{\alpha}\right) \frac{\left(\rho_{n}-\rho_{p}\right)^{2}}{\rho_{0}}
\end{aligned}
$$

where $\rho_{0}=0.16 \mathrm{fm}^{-3}$ is the nuclear saturation density.

The values of the parameters $A=-356.8 \mathrm{MeV}, B=$ $303.9 \mathrm{MeV}, \alpha=1 / 6, C_{1}=125 \mathrm{MeV}, C_{2}=93.5 \mathrm{MeV}$ are adjusted to reproduce the saturation properties of symmetric nuclear matter and the symmetry energy coefficient. We also extend the discussion to finite temperatures 15.

We first focus on the low density region, where phase transitions of liquid-gas type are expected to be seen in fragmentation events. Since $a, b>0$ and $c<0$, in the case of the liquid-gas phase transition in asymmetric nuclear matter we deal only with instability against isoscalar-like fluctuations as it happens for symmetric nuclear matter. However, at variance with the latter case, now the instability can manifest either as chemical or mechanical depending on the relative strength of the interactions in the system, as discussed previously.

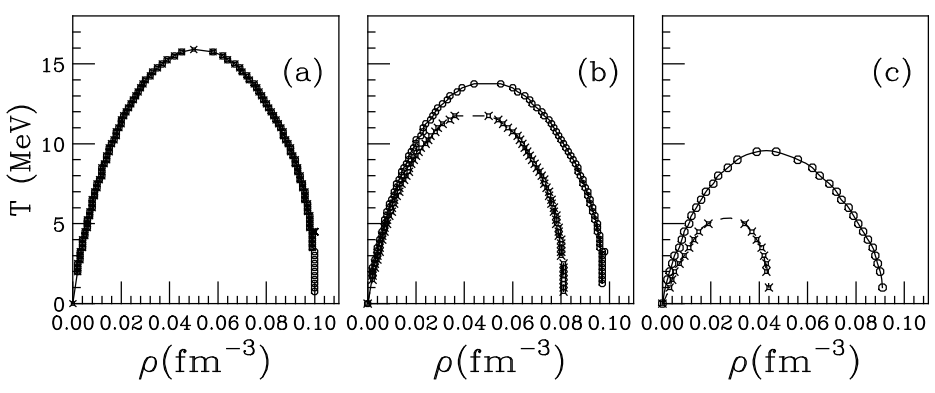

FIG. 1. Spinodal line corresponding to isoscalar-like instability of asymmetric nuclear matter (circles) and mechanical instability (crosses) for three proton fractions: $y=0.5$ (a), $y=0.25$ (b), $y=0.1$ (c).

In Figure 1 the circles represent the spinodal line corresponding to isoscalar-like instability, as defined above, for three values of the proton fraction. For $y<0.5$ under this border one encounters either chemical instability, in the region between the two lines, or mechanical instability, under the inner line (crosses). The latter is defined by the set of values $(\rho, T)$ for which $\left(\frac{\partial P}{\partial \rho}\right)_{T, y}=0$. We observe that the line defining chemical instability is more robust against the variation of the proton fraction in comparison to that defining mechanical instability: reducing the proton fraction it becomes energetically less and less favoured to break in clusters with the same initial asymmetry.

From Eq.s(16) and (17), we see that the quantity $\left(\frac{\partial \mu_{p}}{\partial y}\right)_{T, P}$ changes the sign passing through zero when we cross the spinodal line corresponding to chemical instability and passing through infinity when we cross the line associated with mechanical instability. However, from Eq.(16) (see also Fig. 3), we get that at the inner spinodal line, the ratio $\left(\frac{\partial \mu_{p}}{\partial y}\right)_{T, P} /\left(\frac{\partial \rho}{\partial P}\right)_{T, y} \propto X Y$ is a finite negative number. Therefore we conclude that the singularities of two quantities cancel by taking the ratio. Note also the smooth behaviour of the mixing angle $\beta$ through the mechanical instability line, Fig.2a.
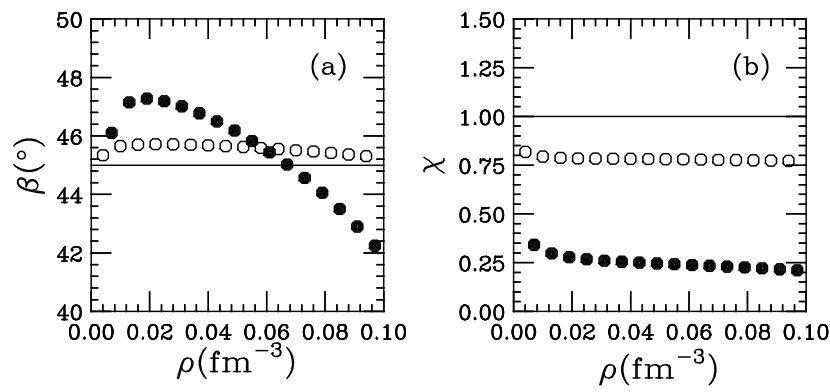
FIG. 2. Density dependence of the mixing angle $\beta$, Eq.(9), (a) and of the function $\chi$ (b) for three proton fractions, $y=0.5$ (solid), $y=0.4$ (open circles), and $y=0.1$ (full circles) at $\mathrm{T}=1 \mathrm{MeV}$.

Finally let us stress that, for $A N M$, even if we deal with instabilities against isoscalar-like fluctuations, we encounter a chemical effect, which gives raise to isospin distillation in equilibrium phase transitions [11]. Indeed the variation of the asymmetry $(I=1-2 y)$ is

$$
\delta I=\nu_{p}\left[\frac{\left(1-I_{0}\right) N_{n}}{\left(1+I_{0}\right) N_{p}} \operatorname{tg} \beta-1\right] \equiv \nu_{p}[\chi-1]
$$

where $I_{0}$ is the initial asymmetry. For $y=0.5$ we have $\delta I=0$ but for $y<0.5$, we find $\chi<1$ (see Fig. $2 \mathrm{~b}$ ). Therefore $\delta I<0$ if $\nu_{p}>0$ and the opposite for $\nu_{p}<0$.

To be more general, we have extended our investigation also to the high density region. In Fig.3 we plot the density dependence of the generalized Landau parameters. We find that for the considered interaction the system exhibits another instability at high density, around $1.5 \mathrm{fm}^{-3}$, where the quantity $c$ becomes positive and $1+F_{0 g}^{a}<0$ (or $\left.Y<0\right)$.
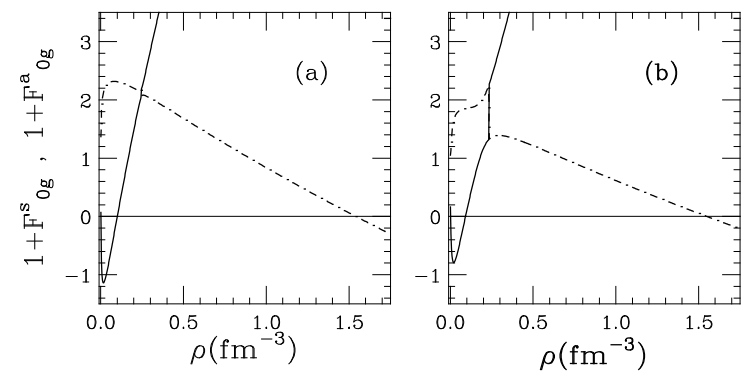

FIG. 3. Density dependence of the generalized Landau parameters for two proton fraction, $y=0.4$ (a) and $y=0.1$ (b) (symmetric, solid and antisymmetric, dashed) at $\mathrm{T}=1 \mathrm{MeV}$.

Thermodynamically we see from Eq.(16) that this is again a chemical instability. However now it results from isovector-like fluctuations, in contrast to the low density instability. The reason is the change in the character of the interaction between the two components. Since the interaction becomes repulsive the nuclear phase can become unstable against proton-neutron separation. We also notice in Fig.3 that the generalized Landau parameters display a discontinuity where the quantity $c$ changes the sign. Other effective forces, with more repulsive symmetry terms, will not show this high density chemical instability [17], that actually could be of interest for other many body systems.

In conclusion, we have shown that in asymmetric binary systems the relevant instability regions are defined by the instabilities against isoscalar-like and isovectorlike fluctuations. The kind of thermodynamical instability, chemical or mechanical, will depend on the relative strength between the various interactions acting in the system.

In particular, the liquid-gas phase transition in asymmetric nuclear matter results from instability against isoscalar-like modes rather than isovector-like due to the attractive character of the interaction between protons and neutrons. This is a qualitatively new effect which is leading to the observed "isospin distillation" [11]. More data are expected from the new Radioactive Beam facilities opening the possibility of direct studies of the charge dependent part of the nuclear interaction far from normal conditions.

[1] G.Sauer, H.Chandra and U.Mosel, Nucl.Phys. 264221 (1976).

[2] H.R.Jaqaman, A.Z.Mekjian and L.Zamick, Phys.Rev. C29 2067 (1984).

[3] G.F.Bertsch and P.J.Siemens, Phys. Lett. 126B 9 (1983).

[4] C.J. Pethick and D.G. Ravenhall, Nucl. Phys. A $47119 \mathrm{c}$ (1987).

[5] H. Heiselberg, C.J. Pethick and D.G. Ravenhall, Phys. Rev. Lett. 61818 (1988).

[6] M. Colonna and Ph. Chomaz, Phys. Rev. C 491908 (1994).

[7] J.D.Frankland, B.Borderie, M.Colonna and INDRA Collab., arXiv:nucl-ex/0007020, Nucl.Phys. A, in press.

[8] H. Müller and B. D. Serot, Phys. Rev. C 522072 (1995).

[9] Bao-An Li, C.M. Ko, Nucl. Phys. A 618498 (1997).

[10] V.Baran, M.Colonna, M.Di Toro and A.B.Larionov Nucl. Phys. A 632287 (1998).

[11] H.S.Xu et al., Phys.Rev.Lett. 85716 (2000)

[12] G. Baym and C. J. Pethick in The physics of Liquid and Solid Helium edited by K.H. Bennemann and J.B. Ketterson, Vol 2, (Wiley, New-York, 1978), p.1.

[13] C.J. Pethick and D.G. Ravenhall, Ann. Phys. (N.Y.) 183 131 (1988).

[14] L.D. Landau and E.M. Lifshitz, Statistical Physics, (Pergamonn Press, 1989), p. 288.

[15] H. Heiselberg, C.J. Pethick and D.G. Ravenhall, Ann. Phys. (N.Y.) 22337 (1993).

[16] The extension of the formalism to momentum dependent interactions is straightforward: see [12] or 17] for an approach based on kinetic Landau equations.

[17] M. Colonna, M. Di Toro and A.B. Larionov, Phys. Lett. 428B 1 (1998).

[18] The necessary stability condition $X Y \propto\left(1+F_{0}^{n n}\right)(1+$ $\left.F_{0}^{p p}\right)-F_{0}^{n p} F_{0}^{p n}>0$ is found also following a kinetic approach based on Landau-Vlasov equation, see [10.17. 